\title{
Las ciudades en la gobernanza global multinivel: una aproximación teórica*
}

\author{
Cities within Multilevel Global Governance: \\ A Theoretical Approximation
}

\author{
Ray Freddy Lara Pacheco ${ }^{* *}$ \\ Centro Universitario de Ciencias Económico-Administrativas (CUCEA), \\ Universidad de Guadalajara \\ Olga María Cerqueira Torres ${ }^{\star * *}$ \\ Centrum Católica Graduate Business School, \\ Pontificia Universidad Católica del Perú
}

Recibido: 03 de marzo de 2017. Aprobado: 09 de septiembre de 2017.

\begin{abstract}
Resumen
La actuación de las ciudades como fuerza política eficaz e influyente en la gobernanza global multinivel cobra cada vez más relevancia a partir del nuevo milenio. Una de las manifestaciones del potencial de la ciudad está, por ejemplo, en la provisión de soluciones a problemas locales que pueden ser escalables globalmente, y viceversa. De ahí el oportuno planteamiento de una agenda de investigación más allá de las establecidas por la paradiplomacia y la ciudad global que identifique categorías/variables clave que ayuden en la propuesta de trabajos de corte empírico, como puede ser la imagen, la

* El presente artículo es resultado del proyecto "La inserción en el medio internacional de las ciudades modelo y sus políticas públicas", perteneciente al Cuerpo Académico en Creación "Las relaciones internacionales y las nuevas diplomacias”. Convocatoria 2017, Programa para el Desarrollo Profesional Docente, para el Tipo Superior (PRODEP), México.

** Doctor en Estudios Internacionales por la Universidad del País Vasco. Centro Universitario de Ciencias Económico-Administrativas (CUCEA), Departamento de Políticas Públicas, Universidad de Guadalajara. ray. lara@cucea.udg.mx

*** Doctora en Relaciones Internacionales por la Universidad Complutense de Madrid. Centrum Católica Graduate Business School, Departamento de Marketing, Emprendimiento y Responsabilidad Social, Pontificia Universidad Católica del Perú. ocerqueira@pucp.pe
\end{abstract}


agencia e identidad, tan necesarios para asentar y confirmar los presupuestos en torno a la ciudad en la gobernanza global multinivel.

Palabras clave: gobernanza global, paradiplomacia, ciudades globales.

\begin{abstract}
The performance of cities as an efficient and influential political force in multilevel global governance is becoming more and more relevant in this millennia; for example, in the provision of solutions to local problems that can be tailored globally, and vice-a-versa. This is one of the manifestations of the potential of cities within global governance, and from there the opportune approach of an agenda of investigation of the context of the city in relation to its role in the global context beyond the Paradiplomacy and the Global City approaches. That in turn implies an identification and analysis of categories of key investigation such as: image, agency, and identity, with the objective to generate not only points of debate, but more importantly a theoretical proposal that later supports the approach of empirical work that is necessary to set and confirm the budgets of the city within the context of multilevel global governance.
\end{abstract}

Keywords: global governance, paradiplomacy, global cities.

\title{
1.- Introducción
}

La reconfiguración del Estado-nación

implica no sólo un cambio en la percepción de la sociedad internacional, desde una sociedad de poderes encontrados hasta una sociedad global con problemas y amenazas globales, que desbordan las políticas nacionales, sino que obliga a los actores, estatales o no estatales, a adoptar políticas comunes y globales y a plantearse la necesidad de la gobernanza global. (Del Arenal, 2009, p. 247)

44 Si Somos Americanos. Revista de Estudios Transfronterizos 
El concepto de gobernanza está siendo utilizado en diversas subdisciplinas de las ciencias sociales (CC. SS.) ${ }^{1}$, "su éxito aparente del concepto parece ser que reside en que refleja la necesidad social de nuevas iniciativas basadas en la realización de crecientes interdependencias sociales" (Kooiman, 2004, p. 171).

La gobernanza global es uno de los ejes de estudio centrales en las relaciones internacionales (RR.II.) de inicios del siglo XXI, eso lo demuestra la diversidad de estudios que se han hecho del fenómeno. Así, "la narrativa que ha surgido sobre la gobernanza global plantea nuevas preguntas sobre la práctica de la soberanía y la autoridad en el mundo de hoy" (Amen, McCarney, Toly y Segbers, 2011, p. 2). Thomas J. Biersteker opina que:

Aunque el ámbito de la gobernanza global, predominantemente ha sido ocupado por los Estados y los Organismos Intergubernamentales, existe una variedad de actores institucionales, específicamente los Actores No Estatales, los cuales tienen un papel más relevante en la gobernanza global contemporánea. Ya que articulan formas alternativas de gobernanza, desempeñan un papel activo en la formulación de la agenda, además de que crean espacios con la intención de proponer ciertas normas que puede ser establecida a nivel general. Durante mucho tiempo estuvieron ausentes debido al tipo de gobernanza establecido por la sociedad internacional de los Estados, y en gran medida invisible para la hegemonía estatal, pero actualmente son los jugadores principales en la producción de las normas internacionales así como en el ámbito de la autoridad privada. (Biersteker, 2009, p. 22) ${ }^{2}$

El enfoque de la gobernanza global multinivel plantea que el sistema está constituido por redes de múltiples actores (estatales y no estatales) y que interactúan a través de niveles, como la fuerza política más eficaz e influyente en el gobierno global (Forman y Segaar, 2006, citados en Simmerl, 2011, p. 23). En este marco, la posibilidad por parte de las ciudades de elaborar estrategias de inserción internacional incrementa sus opciones de mejorar su desempeño en torno a sus procesos de gobernanza urbana y global. Es por ello que el presente artículo tiene como objetivo señalar la importancia del enfoque

\footnotetext{
"En el mundo anglosajón, governance es una palabra que ha sido utilizada habitualmente a lo largo de siglos para referirse al ejercicio de la autoridad dentro de una determinada esfera" (Hewitt, 1998, p. 1). La "gobernanza es una palabra y un concepto que se ha vuelto últimamente muy popular. Durante mucho tiempo, la palabra 'gobernanza' significó simplemente 'gobernar', y esto referido al aspecto del proceso de gobierno. Sin embargo, en la actualidad el término se utiliza, sobre todo, para indicar un nuevo modo de gobernar" (Mayntz, 2001, p. 1).

2 Para T. Biersteker (2009), la mayoría de los estudios sobre la gobernanza global centran su análisis en las bases de la gobernanza: 1) sociedad de estados; 2) hegemonía; 3) regímenes internacionales; 4) instituciones; 5) derecho internacional; 6) normas globales; 7) autoridad privada. Para él, gran parte de la literatura académica en las RR.II. está dedicada a los debates sobre estas bases, sin ir más allá.
} 
de la ciudad global y sus implicaciones en las acciones de cooperación transfronteriza, inserción internacional (imagen e identidad), y agencia en el sistema internacional de los gobiernos locales, más allá de la paradiplomacia como campo de estudio desde las relaciones internacionales.

\section{2.- Los actores en la gobernanza global multinivel}

Una característica fundamental que define al sistema de gobernanza global multinivel es la multiplicidad de los actores y su interacción. Desde una perspectiva constructivista, Georg Simmerl (2011) sostiene que "una multitud de actores ha emergido [...] y se involucran en conjunto con los representantes de los Estados en luchas discursivas sobre como gobernar el globo" (p. 18). En esta línea, señala tres dinámicas que desarrollan los actores dentro del sistema de gobernanza, y que son esenciales para entender la estabilidad y el cambio en el orden mundial, estas son: 1) formación discursiva; 2) hegemonía, y 3) lucha discursiva.

A través de la formación discursiva se tiene una red de conceptos en textos y lenguaje hablado que los actores del sistema tienen que rearticular en orden a participar exitosamente en los discursos de un área específica. La hegemonía es la forma temporal de interpretar las formaciones discursivas en diferentes áreas y compartidas por una mayoría de actores, que asegura la cooperación y el consentimiento de estos en diferentes niveles de la sociedad mundial. Mientras que la lucha discursiva puede ser entendida como la interacción entre los actores políticos por las interpretaciones dominantes de los sucesos políticos y las políticas adecuadas en campos estructurados por formaciones discursivas específicas ${ }^{3}$.

Siguiendo esta lógica, se puede establecer entonces que, de los procesos comunicativos entre los actores estatales y no estatales - en esta categoría se incluyen a las organizaciones no gubernamentales, las organizaciones internacionales, las entidades subestatales,

\footnotetext{
3 Por poner un ejemplo de esta dinámica, según Simmerl (2011), "una intervención humanitaria sólo es posible si una multitud de actores estatales y no estatales está unida por un entendimiento fundacional hegemónico de la superioridad de la seguridad humana cuya protección es una razón legítima para infringir la soberanía nacional. En este ejemplo, las formaciones discursivas estarían conformadas por los términos 'seguridad humana', 'soberanía nacional', '(no) intervención'; mientras que la hegemonía por el entendimiento de una multitud de actores estatales y no estatales sobre la conveniencia o no de la intervención, y la lucha discursiva se daría a través del proceso para llegar a dicho entendimiento" (p. 10).
} 
las corporaciones trasnacionales, las universidades, las comunidades epistémicas, los movimientos sociales y las redes trasnacionales de cabildeo e influencia- se producen las interpretaciones dominantes de los sucesos políticos y las políticas en el marco de la gobernanza global multinivel.

Estos procesos comunicativos, según David Criekemans,

implican el desarrollo de actividades diplomáticas desplegadas por una red de actores que cada vez se torna más condensada: la diplomacia está cambiando rápidamente tanto de naturaleza como de contenido. A través de una descentralización de las relaciones internacionales, que podría afectar el entendimiento convencional de la diplomacia como un dominio exclusivo de los Estados soberanos. (Cornago, 2010, p. 12)

No obstante, "los representantes de los Estados aún retienen las posiciones de interlocución más fuertes, pues aún tienen a cargo la mayor parte de la autoridad formal en la gobernanza global" (Simmerl, 2011, p. 19). Por tanto, los Estados son aún los principales actores del sistema, aunque Alexander Wendt considera que también puede haber otros actores (citado en Durán, 2013, p. 186), los cuales van incrementando su participación, como pueden ser las ciudades.

\section{3.- Los actores no estatales y la paradiplomacia en la gobernanza global multinivel}

La participación de los actores no estatales en la gobernanza global se traduce en nuevos desafíos de cara a la determinación de la subjetividad internacional, pues sujetos de las relaciones internacionales evidencian procesos de comunicación cada vez más formales con sujetos del Derecho Internacional Público (en este caso los Estados soberanos). Procesos de comunicación que revelarían que la actuación desplegada por los actores no estatales a nivel global en un plano sociológico tienen también una creciente repercusión en el plano jurídico, en un contexto de "una progresiva tendencia al ensanchamiento del 
círculo de sujetos del Derecho Internacional Público [...] pues las propias necesidades de la comunidad jurídica internacional pueden aconsejar o incluso exigir el investir de personalidad internacional a determinadas entidades" (Diez de Velasco, 2013, p. 275).

Se hace la diferenciación de los planos sociológico y jurídico, entre otras cosas, por el alcance de los acuerdos en ambos planos pues, cuando se habla de relaciones en el plano sociológico, la efectividad de los acuerdos que se adoptan entre los actores dependerá exclusivamente de la eficacia de los mecanismos que hayan previsto entre los contrayentes para concretar dicho acuerdo. En cambio, cuando se trata de acuerdos contraídos en el plano jurídico (donde los Estados son sujetos de derecho originarios) existe el ordenamiento internacional, el Derecho Internacional, para garantizar el cumplimiento de dichos acuerdos.

Los actores no estatales no ostentan de manera formal esa condición de sujeto del Derecho Internacional. Sin embargo, se puede sostener de forma más recurrente que estos actores manifiestan una aptitud para hacer valer su condición de sujetos ante instancias internacionales. Es decir, si bien no son titulares de derechos y obligaciones conferidos por normas jurídicas internacionales, sí apuntan a uno de los elementos (aptitud de condición de sujetos) que conforman la doble exigencia en el plano procesal de la legitimación como sujetos.

Sin embargo, no es suficiente para determinar una personalidad internacional objetiva, cuya extensión es obra de los Estados, "los cuales pueden actuar bajo la presión de las fuerzas sociales (nacionales o internacionales)" (Truyol, citado en Diez de Velasco, 2013, p. 278), por lo que el alcance del discurso de estos actores (no estatales) en los procesos comunicativos genera cambios en la(s) estructura(s) en la(s) que se desenvuelven y en su propia estructura, la perspectiva principal que ha analizado este fenómeno es conocido en los círculos académicos como paradiplomacia ${ }^{4}$.

Stéphane Paquin (2005) comenta que "en política comparada y en las relaciones internacionales a menudo se minimiza la importancia de la paradiplomacia, ya que los actores carecen de las cualidades requeridas para ser consideradas como 'verdaderos protagonistas internacionales"' (p. 129). La Paradiplomacia — con mayúsculas - ha sido uno de los campos de estudio de acogida de estos actores y sus relaciones, y el que más

$4 \quad$ El concepto surgió en el artículo de Butler (1961). "Ivo Duchacek (1986) revivió la idea de paradiplomacia como una forma de agencia política de las entidades subnacionales. Donde éstos perforan la soberanía (Duchacek, Latouche y Stevenson, 1988) de los Estados, priorizando sus intereses particulares a través de conexiones transfronterizas, transregionales y globales" (Acuto, 2013a, p. 8). 
ha revisado la dinámica de estos entes a nivel internacional, debido a que "podría ayudar a que la teoría de las RR. II. vaya asimilando el cambio, incorporando nuevos actores como los gobiernos locales en sus conceptos" (Marx, 2010, p. 39). Entendiendo que "la diplomacia sub-estatal difiere de otras formas históricas y contemporáneas de diplomacia de actores no estatales en virtud de su disposición territorial" (Duran, 2013, p. 63).

A finales de los ochenta y principios de los noventa, la paradiplomacia, entendida como diplomacia paralela (parallel diplomacy), comenzaba a expandirse en varias partes del mundo - en especial en los países con Estado federal-. Ivo Duchacek señalaba lo siguiente:

La imagen que se obtenía, era que en el ámbito internacional no solo se escuchaba la voz de los Gobiernos Centrales sino también la de otros protagonistas como la oposición, las comunidades etno-territoriales, los grupos de interés, las partes componentes de los estados (provincias, municipios o sus equivalentes) etc., de manera que resultaba una suerte de polifonía, de voces multívocas, prestando especial atención analítica a los actores gubernamentales subnacionales, a los cuales más tarde terminaría incorporando entre los entes perforadores de la soberanía estatal. (Duchacek, 1986, citado por Luna, 2010, p. 55)

Dentro de los Estados federales y descentralizados, en palabras de Duchacek, quien fue él que diseminó el concepto de paradiplomacia en la literatura académica, la define como: "Las intervenciones de los gobiernos no centrales en las relaciones internacionales" (2010, p. 38), la cual podría entenderse como las actividades transoberanas, las actividades internacionales y las actividades transgubernamentales de los gobiernos no centrales (GNC) con sus contrapartes transfronterizas, transregionales y globales. Por su parte, Panayotis Soldatos la define como "la política exterior de los gobiernos no centrales" (citado en Criekemans, 2010, p. 62), “apoyando, complementando, corrigiendo, duplicando o desafiando la diplomacia del estado-nación" (citado en Bursens y Deforche, 2010, p. 155).

En este contexto, las unidades subnacionales quedaban definitivamente asumidas como actores relevantes de la política mundial y las relaciones internacionales generadas desde esa escala comienzan a recibir un tratamiento específico y pormenorizado, favorecido por la emergencia de una nueva retórica acerca de lo local y de perspectivas que revisan las concepciones tradicionales de espacialidad y territorialidad que habían limitado la perspectiva disciplinaria de las relaciones internacionales y de las ciencias sociales en general (Pont, 2010, pp. 55-56). 
Para Soldatos (1990), las acciones paradiplomáticas pueden variar de naturaleza, por un lado, pueden ser acciones de cooperación (o apoyo) cuando las actividades por parte de las unidades federadas son coordinadas por el gobierno federal o son desarrolladas de manera conjunta. Por otro lado, son acciones paralelas (o sustitutivas) cuando se desarrollan en un clima armonioso, donde el gobierno federal acepta la racionalidad de la acción independiente de una entidad confederada en las relaciones exteriores, con o sin la supervisión federal. Por otra parte, dicha acción puede estar en conflicto con el gobierno federal, esta última contra un recurso en contenido o forma, a lo que se le llama fragmentación (pp. 38 y 40).

La mayoría de los autores definen la paradiplomacia como las actividades externas del gobierno subnacional/subestatal/no central (Aldecoa y Keating, 1999; Duchacek, 1990; Lecours, 2008; Philippart, 1997;). Éric Philippart (1997) ha clasificado como: mínima, menor, mayor, y máxima paradiplomacia así como la protodiplomacia; donde la paradiplomacia mínima define la cooperación transfronteriza, centrándose en la cooperación socioeconómica y cultural, mientras que en el otro extremo, la diplomacia máxima define a un fuerte regionalismo y nacionalismo, y tiene por objeto reforzar los beneficios regionales, y la protodiplomacia es la fase transitoria hacia la independencia.

Alexander Kuznetsov señala la importancia actual en la cuestión práctica y teórica de este campo de estudio. Con relación a este último punto, identifica las once dimensiones para el estudio de la paradiplomacia: 1) constitucional; 2) federalista y relaciones intergubernamentales; 3) nacionalismo; 4) relaciones internacionales; 5) estudios de frontera; 6) globalización; 7) seguridad y geopolítica; 8) economía global; 9) medio ambiente; 10) diplomacia; 11) separatismo. Así, la importancia de este subcampo de conocimiento de las RR.II. se entiende en los siguientes términos ${ }^{5}$ :

Es uno de los aspectos del cambio sistémico dentro del entorno internacional. Que pasó de ser un conglomerado de unidades Estatales a un mundo de coexistencia de actores estatales y no estatales. Se ha convertido en un mini-laboratorio para la diplomacia experimental, como resultado de las discrepancias jurídico-internacionales y constitucionales entre el Estado y la diplomacia no estatal. (Duran, 2012, p. 5)

$5 \quad$ Revisar Kuznetsov (2015). 


\section{1.- Críticas a la paradiplomacia}

La perspectiva teórico-metodológica de este subcampo, así como el término paradiplomacia, no están exentos de controversia, aunque este último es aún el más usado. Precisamente por su amplitud, ya que sirve de protección y/o amparo - por tanto, efecto paraguas-a las diversas situaciones descritas líneas arriba: apoyando, complementando, corrigiendo, duplicando o desafiando la diplomacia del Estado-nación. Se señalan las cuatro más importantes, las cuales están íntimamente relacionadas: 1) el término y/o definición del concepto; 2) el sujeto y/o objeto de estudio; 3) el tipo de actividades que se realizan en el sistema internacional por parte de estos actores; 4) la agenda de investigación y/o estudio; 5) la agencia de los actores no estatales.

\subsubsection{El término y/o definición del concepto}

Si se asume un criterio práctico, se concluirá que el término paradiplomacia aún resulta el más usado debido a su amplitud para incluir todos los perfiles proyectados por los actores no estatales y los distintos gobiernos subnacionales/subestatales/no centrales en el mundo. En opinión de Brian Hocking (1993), "el término presume implícitamente intereses incompatibles" (citado en Criekemans, 2010, p. 62), ya que esto puede generar rechazo por parte de ciertos actores que participan u observan, ya sea como políticos, técnicos, académicos, ciudadanos. Debido a ello, propone el término "Diplomacia de Múltiples Capas/Niveles (Multilayered Diplomacy)" a través del que describe las vinculaciones y complementariedades entre la diplomacia estatal y no estatal.

Rogier van der Pluijm y Jan Melissen (2007) consideran el término paradiplomacia como desafortunado e inapropiado, apuntando más bien hacia la complementariedad entre ambas diplomacias, la estatal y no estatal. Teniendo en cuenta que,

La diplomacia es vista como una red de interacciones cambiantes entre distintos actores, en función del ámbito de la política, los intereses y la capacidad de operar en un ambiente determinado. De este modo, las entidades sub-estatales no están solamente cambiando las reglas de la diplomacia, al permitir que no sólo los estados sean los que actúan, sino también naturaleza de la actuación mediante la alteración de las relaciones entre los diferentes actores, dejando más espacio para los patrones flexibles, fluidos e improvisados en la diplomacia. En esta nueva diplomacia catalítica, las regiones se convierten en constructores de puentes y guardianes dentro de una 
matriz diplomática cada vez más compleja que abarca todo tipo de actores en todo el mundo. (Hocking, citado en Durán, 2013, p. 61) ${ }^{6}$

Se puede apreciar, que el término “'Para' transmite la idea de estar ante rutas diplomáticas paralelas, y la interrogante que se puede plantear al respecto es si el gobierno subnacional/sub-estatal/no central puede desarrollar una ruta diplomática paralela a la del Estado central" (Van der Pluijm y Melissen, 2007, p. 9). Sin profundizar demasiado en la reflexión, la respuesta es no. Ejemplo de ello es la situación propia en América Latina:

Más allá de los contornos territoriales inmediatos de esta realidad, el nuevo impulso a la participación de gobiernos sub-estatales en asuntos internacionales en los países latinoamericanos se desarrolló también en sintonía con las reclamaciones de un reconocimiento institucional y político mayor de las entidades sub-estatales, y las reivindicaciones de un control democrático más efectivo de la formulación de políticas tanto en asuntos internacionales como domésticos. (Cornago, 2016, p. 27)

Estas visiones promovidas por Hocking (1993), Van der Pluijm y Melissen (2007) señalan que:

La diplomacia de múltiples capas/niveles se erige como una nueva alternativa a la paradiplomacia. El enfoque subraya que la gama de actores e intereses en el proceso de construcción de la política exterior es muy diversa y estos actores están todos involucrados en el logro de un complejo conjunto de acuerdos transfronterizos en el marco de las políticas globales (Amen et al., 2011).

Para otros especialistas en la materia, se observan dos razones que justifican el interés por la denominación "diplomacia sub-estatal" - entendida como las actividades externas de los meso gobiernos y los grupos de interés que interactúan dentro de sus territorios-. La primera es que encaja dentro de denominaciones más amplias, que denotan consenso, como la de diplomacia de múltiples capas/niveles, y la segunda es que resulta un término preciso para identificar el nivel de análisis que se quiere investigar, la diplomacia desplegada por el gobierno subestatal, puesto que esta categoría contiene a la diplomacia del gobierno local en el proceso de internacionalización y cooperación y transfronteriza, y como lo hacen notar Gilberto Aranda, Cristian Ovando y Alejandro Corder (2010):

6 Sobre la genealogía de la diplomacia, revisar Der Derian (1987). 
Conjeturamos que sus notas distintivas, que nos permiten establecer sus significados posibles y atributos, están suficientemente desarrolladas, pero su extensión, es decir, el conjunto de casos de referencia, especialmente los casos de este lado del mundo - América Latina-, si bien han empezado a estudiarse, el tratamiento conceptual no ha sido lo suficientemente cotejado, trayendo como consecuencia cierta imprecisión y distorsión de lo que realmente se quiere estudiar con este neologismo. (p. 35)

En esta misma dirección, Criekemans (2010) establece: "Quizá ha llegado el día para dejar al término paradiplomacia descansar, y en su lugar utilizar un término más neutral, diplomacia sub-estatal" (p. 64), "pues el mismo es más un reflejo del nivel de análisis en el que se desenvuelve este tipo de diplomacia" (Duran, 2013, p. 29). Estos enfoques tienden a enfatizar las dimensiones consensuadas e incluyentes de esta realidad, sobre sus posibles aspectos controvertidos, sostiene Cornago (2010):

No obstante, y con el fin de evitar posibles disputas terminológicas, la diplomacia subestatal puede quizás ser una denominación más apropiada, para una realidad que se está convirtiendo en un lugar común en los procesos de formulación de políticas cotidianas de muchos gobiernos locales y regionales en todo el mundo y es cada vez más aceptado por el propio sistema diplomático. (p. 20)

\subsubsection{El sujeto y/o objeto de estudio}

Aunque de esta segunda razón del uso de diplomacia subestatal proviene el siguiente cuestionamiento, y que presentan los estudios paradiplomáticos desde hace treinta años, es lo que se estudia: ¿la denominación de lo que se analiza (objeto de investigación) es el territorio (provincia, región, Länder, municipio, ciudad) como un todo, o son los gobiernos de esos territorios los verdaderos actores internacionales? La respuesta sería que uno de los grandes errores que se observan en los estudios paradiplomáticos es la agrupación en una sola vasija de la infinidad de formas existentes de gobiernos no centrales, como los actores subnacionales o los subestatales, y en menor medida, las ciudades, los municipios, etc., sin incluir otro tipo de actores no estatales. Teniendo claro lo siguiente:

En el modo de denominación del "nuevo" actor: suele mencionárselas como unidades o entidades subnacionales, gobiernos no centrales, unidades subestatales, unidades infraestatales, actores territoriales, meso-gobiernos, entre otros; denominaciones que reflejan distintos criterios o ángulos de observación: en términos jurídicos, funcionales, de la relación jerárquica con el Estado, y aún el tipo de régimen político o de configuración de la nacionalidad. (Pont, 2011, p. 69) 
Donde

el carácter territorial de la unidades, constituye uno de los tres atributos de los que están dotadas estas entidades sub-estatales, y que corresponden a atributos del Estado. Los otros dos son: la población que vive en este territorio, y un gobierno con un poder legislativo y ejecutivo. La soberanía, la cuarta dimensión central del Estado, es (todavía) de la que carecen las entidades sub-estatales, aunque esto es reclamado por algunas entidades subnacionales con fuertes tendencias nacionalistas como Quebec, Cataluña o el País Vasco (Therney, 2005, citado en Durán, 2013, p. 46).

Lo precedente reforzaría la posición neorrealista, en cuanto a que se requiere de atributos propios del Estado para legitimar y dar relevancia a la actuación diplomática de los gobiernos subestatales.

Mónica Salomón (2011) sostiene lo siguiente:

Nuestro objeto de estudio es la acción exterior de los gobiernos subnacionales, no la de las regiones o entidades subnacionales [...] ni ningún otro actor basado en ellos, por ejemplo, las universidades o empresas, no obstante éstas pueden desarrollar sus relaciones internacionales. (p. 4)

Esta visión limita las relaciones entre estos actores con los gobiernos locales con miras a concretar proyectos conjuntos de inserción del territorio en donde están asentados. Esto lo hace para justificar el grado exploratorio de su proyecto de investigación en Brasil, centrado solo en los gobiernos (regionales: gobiernos estaduales; y locales: prefecturas municipales).

Para empezar, no está de más insistir en que nuestro objeto de estudio es la acción exterior de los gobiernos subnacionales, no la de las "regiones" o "ciudades" o "entidades subnacionales", términos que aparecen frecuentemente en la literatura sobre nuestro tema. La tendencia a usar esos términos como intercambiables con el de gobierno local o regional es, de cierto modo, natural. (Salomón, 2007, p. 2)

Como se sabe, normativamente hablando, no es lo mismo una provincia que una comunidad autónoma, región, entidad federativa, Länder, cantón, cada una de ellas tiene una estructura jurídico-legal propia; y desde una perspectiva más subjetiva, una identidad moldeada por los distintos actores que se encuentran dentro de ellas. Así, las 
entidades subestatales o subnacionales "pueden ser definidas como 'una entidad territorial coherente situada entre los niveles nacional y local, con una capacidad y autoridad para la toma de decisiones'” (Hooghe y Schakel, 2008, p. 113, citados por Duran, 2013, p. 47 ), lo que deja en duda otras formas legales como el mismo municipio, los gobiernos metropolitanos y la misma ciudad.

3.1.3. El tipo de actividades que se realizan en el sistema internacional por parte de estos actores

A diferencia de los gobiernos centrales (que representan a los Estados):

Los gobiernos locales pueden voluntaria y discrecionalmente tener relaciones internacionales o no tenerlas. Uno de los grandes retos que afrontan es que su actuación ha estado sujeta en gran medida a la prueba y el error o a las vicisitudes políticas del momento. (Zapata, 2007, p. 18, citado en Lara, 2015, p. 144)

"Sin que éstas se enmarquen en un planteamiento estratégico, ni estén concebidas de manera integral. Emprendidas, en general, a iniciativa de los diferentes servicios y departamentos o que responden simplemente a ciertas demandas procedentes del exterior" (Malé, Cors y Sanz, 2014, pp. 49 y 51). De hecho, uno de los mayores propósitos en la consolidación de la diplomacia subestatal consiste en que el gobierno local desarrolle una serie de políticas públicas estratégicas y comprehensivas, que resulten esenciales en su proceso de comunicación en el contexto global.

La realidad indica que ha sido el conjunto de prácticas diplomáticas tradicionales, pero también otras acciones las que han hecho posible las relaciones exteriores de estos actores. Y esto es porque cuando estudiamos a la paradiplomacia, el objeto de estudio en observación no es la actividad de un Estado (el cual es sujeto de Derecho Internacional Público), a través de su gobierno. Así resulta entendible identificar diferencias entre diplomacia y política exterior cuando se habla de Estados y cuando se habla de actores subestatales, no estamos ante los mismos actores. Por lo que sus procesos de comunicación en el contexto global no pueden ser definidos bajo el mismo prisma. Es por ello, y como lo hace notar Mariana Luna Pont (2011), que:

Difieren las formas de encuadrar su desempeño: entendido como expresión de gestión/acción/actividad/proyección exterior; como una variante particular de relaciones internacionales - relaciones internacionales subnacionales-; como formas alternativas de diplomacia - paradiplomacia, diplomacia subnacional, diplomacia 
constituyente, diplomacia multinivel, postdiplomacia, etc.-; como un tipo particular de política exterior - política exterior descentralizada, política exterior federativa- $y$, más recientemente, como expresión de la emergencia de nuevas formas de gobernanza (governance). (p. 69)

Caterina García i Segura (1996) señala las primeras grandes cuestiones que surgen en torno a ello,

Desde la perspectiva de las RR. II., hay dos cuestiones que se debaten alrededor de la naturaleza de la actividad exterior subestatal: la primera, perteneciente al terreno puramente teórico, tiene que ver con la categoría de actividad en la que debería incluirse, es decir, si se trata de relaciones intergubernamentales o transnacionales; la segunda, más ligada a la realidad política, se refiere a su relación con la actividad exterior del Estado, a saber, si está o no enfrentada con ésta. (p. 252)

Otro aspecto discutido es el que señala Manuel Duran (2013): "Por alguna razón u otra, las actividades diplomáticas y las externas de estos entes subnacionales son consideradas sinónimas por los autores”, se pregunta si “ies una mera confusión semántica, un desorden terminológico, o hay más de fondo? ¿Hay alguna razón para diferenciar entre la diplomacia y la política exterior cuando se habla de los Estados, al tiempo que esta frontera se hace permeable para las regiones (o sub-estados o entidades sub-nacionales o entidades federales)?” (pp. 26-27). El mismo provee una posible respuesta donde:

Las entidades subnacionales se ven obligadas a improvisar y desarrollar formas híbridas de diplomacia, así la fusión de prácticas diplomáticas tradicionales y alternativas ha permitido el establecimiento de relaciones exteriores, siendo el aspecto territorial, su distintivo frente a otros actores no estatales. (Duran, 2012, p. 5)

\subsubsection{La agenda de investigación y/o estudio}

En trabajos posteriores centrados en los estudios paradiplomáticos y el análisis de políticas exteriores, Salomón (2007) plantea el estado de la cuestión de los estudios de los gobiernos subnacionales en las RR.II.

Dada la relativa novedad del fenómeno (al menos tal como se presenta actualmente) y lo heterogéneo de las situaciones analizadas, resulta natural que la mayor parte de la literatura sobre actores subnacionales sea de carácter descriptivo. Ya hay, sin embargo, 
cierta elaboración conceptual a partir de la cual es posible construir y también desarrollar una agenda de investigación. (p. 2)

Esto lo viene a reafirmar Mariana Luna Pont (2010), al señalar lo siguiente:

Existe una muy abundante literatura que específicamente se ha hecho cargo de la dimensión internacional del desempeño de las unidades subnacionales, sin embargo los esfuerzos para ordenarla sistemáticamente desde la perspectiva del encuadre teórico son más recientes y menos abundantes. Aparece más bien presentada en forma esquemática en el marco de trabajos e investigaciones que se centran en otros aspectos del tema; por otra parte, encontramos numerosos trabajos donde se presentan tratamientos pormenorizados de perspectivas teóricas particulares, pero menos donde se identifican y abordan en forma detallada lo que se consideran las principales aproximaciones teóricas al tema. (p. 51)

Posteriormente, esta misma autora señala que la agenda de investigación de los estudios paradiplomáticos se han construido desde diversas disciplinas de las ciencias sociales, y recientemente observadas desde el campo de las RR.II.:

El accionar internacional de actores subnacionales sean estos municipios, provincias, regiones o sus equivalentes en denominación, es abordado desde diversas disciplinas: las relaciones internacionales, los estudios comparados, la administración pública, los estudios del desarrollo, la economía política internacional, los estudios urbanos, la nueva geografía económica y la nueva geografía política, estudios desarrollados en paralelo pero que, en algunos casos, se retroalimentan y superponen, agregando complejidad a los intentos de sistematizar los modos de tratamiento de esta temática e introduciendo divergencias sustanciales en términos la calificación del actor y la tipificación de su desempeño; la identificación de contextos de surgimiento y factores de activación; las formas de ponderación del fenómeno; el nivel de análisis y las variables explicativas centrales, etc. (Pont, 2011, p. 69)

Desde ese momento, se asume que la agenda de investigación y/o estudio está en construcción y que como todo campo de conocimiento es inacabado, Nahuel Oddone (2016) señala que: "Se pueden registrar cinco grandes encuadres teóricos: 1) Desde el actor internacional; 2) Desde la política exterior y los procesos de toma de decisiones; 3 ) 
Desde el desarrollo territorial; 4) Desde la integración regional; y 5) Desde los enfoques de la gobernanza" (p. 49)

Para fines de este artículo, la observación que se le podría hacer a la Paradiplomacia es que no hace una diferenciación clara entre los enfoques propios de la ciudad y los "enfoques en la cuestión subestatal/subnacional", lo que indica que todo enfoque puede estudiar a la ciudad, y en términos generales así es, pero obvia las características propias de la ciudad frente a otros actores de similares características que hacen diferente su estudio desde las RR. II. Con esta idea, estos entes subnacionales difieren de los municipios, ya sean urbanos o rurales a una metrópoli o una ciudad media.

\subsubsection{La agencia de los actores no estatales}

Para Olga Cerqueira (2013), desde el enfoque constructivista se da énfasis sobre los procesos comunicativos entre los actores estatales, y no estatales, a partir de los cuales se producen las interpretaciones dominantes en el marco de la gobernanza global multinivel, evidenciándose que los intereses e identidades de los diversos actores (estatales y no estatales) no se pueden suponer como fijos, sino son susceptibles de transformación, puesto que sus ideas, identidades e intereses son socialmente construidos y endógenos al proceso de interacción.

Se tiene pues que de los procesos comunicativos se crea y transforma la relación entre los actores, y en particular durante la lucha discursiva se da la interacción entre los mismos por las interpretaciones dominantes de los sucesos políticos y las políticas adecuadas en campos estructurados por formaciones discursivas específicas. Vale decir, el proceso de comunicación/interacción entre los actores va modelando el discurso. Concibiéndose la legitimación de la subjetividad sociológica y jurídica de los actores subestatales como un proceso de formación y lucha discursiva.

\footnotetext{
Dentro de los mismos estudios paradiplomáticos existen dos vertientes claras de estudio, la más normativa y funcionalista (Aldecoa y Keating, 1999; Dávila, Schiavon y Velázquez, 2008; Duchacek, 1984, 1986, 1988, 1990; Fry, 1989, 1998; E. Iglesias, V. Iglesias y Zubelzú, 2008; Jain, 2005; Kaiser, 2003, 2005; Kuznetsov, 2015; Martínez, 2011, 2014; McMillan, 2012; Michelmann, 1989, 1990; Philippart, 1997; Pont, 2010, 2011; Salomón, 1993, 2007, 2011; Smith y Cohn, 1996; Schiavon, 2006; Soldatos, 1989, 1990, 1993; Tuñon, 2008, 2011; Ugalde, 2005, 2006; Velázquez y Ortega, 2014; Villaruel, 2010, 2013, 2016; entre otros) y otra mñas histórico-reflexiva (Aguirre, 1999, 2000; Cornago, 1996, 1999, 2000, 2010, 2013, 2016; Criekemans, 2008, 2010; Der Derian, 1987; González Miranda, 2008, 2014, 2016; Hocking, 1993, 1999; Khanna, 2011; Kincaid, 1999, 2010; Lecours, 2008, 2010; Magone, 2007; Oddone, 2010, 2016; Ovando, 2010, 2013, 2016; Paquin, 2004, 2005, 2010; Rodríguez Gelfenstein, 2004, 2006; Tapia, 2002, 2003; Tierney, 2005; Vigevani, 2006; entre otros).
} 
Así, para Bekemans (2008, p. 3), la gobernanza política implica desarrollar capacidades (institucionales, políticas) para responder rápidamente al cambiante panorama políticoeconómico. Los gobiernos locales no se extraen de estas dinámicas. Así, en la dimensión política de la diplomacia subestatal, la actuación del gobierno local está orientada a la adquisición de estas capacidades.

Con la gobernanza se alude así a un nuevo estilo de gobernar, una modalidad de coordinación no jerárquica, caracterizada por un mayor grado de cooperación, interacción y toma de decisiones entre el Estado y los actores no estatales al interior de redes público-privadas, locales, nacionales o internacionales. (Oddone, 2016, p. 64)

Si bien como lo hace notar Cornago (1996),

La misma proyección internacional de los meso gobiernos, exige precisar además, junto a la dimensión histórica del propio objeto de estudio, tanto las propiedades de los agentes cuya acción nos interesa investigar, como oportunidades y límites que establece para esa acción el contexto siempre cambiante en que tales actores se hallan inmersos. Una cuestión que impone reflexionar sobre la forma en que habremos de conceptualizar, e investigar, en las RR. II. como en cualquier otro dominio de las CC. SS., la relación entre acción y estructura. (p. 33) ${ }^{8}$

Son muy pocos los estudios que revisan la agencia de estos actores en la gobernanza global multinivel, entendiendo la agencia como "capacidad de ser o comportarse como actor" (Grasa, 2000), donde las ciudades en las últimas dos décadas han sido fundamentales en la creación de ciertos regímenes internacionales. A saber,

Los estudios sobre paradiplomacia, han sido capaces de reconocer a las ciudades como actores en los asuntos internacionales, pero carecen del compromiso teórico para explicar: 1) los orígenes de su agencia, y 2) los impactos globales de la creciente interacción entre las ciudades y la gobernanza global. Del mismo modo, un problema clave ha sido el gran desprecio por las fuentes de dicha "agencia externa" de las ciudades, que han aparecido en estos estudios como actores, sin ser problematizada su base interna para asumir su papel en la política mundial. (Acuto, 2013a, p. 9)

Con relación a estos estudios, Michele Acuto señala que el papel diplomático de las ciudades ha recibido hasta la fecha consideraciones muy superficiales. Esta paradiplomacia

8 Sobre el debate de la agencia y estructura, revisar Wendt (1987, pp. 335-370); Wight (2006); Cornago (1996, pp. 21-46). 
solo asume a la ciudad como el locus de las actividades internacionales, y al gobierno de la misma, como el actor funcional que realiza las actividades. Debido a ello, desde esa época hasta ahora han surgido diversos conceptos centrados en las actividades paradiplomáticas de estos lugares y sus gobiernos locales, los cuales han ido abarcando los estudios de la ciudad en el medio internacional.

Las ciudades exceden la proximidad geográfica. De donde resulta dable pensar que lo interesante en el acontecer de las RR. II. no es tanto la perforación de la soberanía como la perforación de los límites, es decir, de todos aquellos obstáculos que han impedido que el hombre pueda sentirse auténticamente libre en su sistema global. De tal manera, no sólo se horadan las fronteras y la soberanía, sino también las cortapisas culturales, los integrismos en materia religiosa, la majestad de ciertas lenguas, la superioridad racial, el sentido de autoridad científica. Se horadan los límites. (Torrijos, 2009, p. 366)

\section{La ciudad global en la gobernanza global multinivel}

De acuerdo con el enfoque de gobernanza global multinivel $-\mathrm{y}$ como se estableció al principio del documento-, es el enfoque que sirve de base para el planteamiento sobre cómo se da la interacción (procesos de comunicación de corte vertical y horizontal) de los actores en el contexto global (entre ellos el gobierno local, la iniciativa privada y la sociedad civil organizada), los asuntos/affairs se gestionan en tres dimensiones: política, económica y social (Bekemans, 2008, pp. 2-3).

Es en esta nueva década (2010-2017) cuando se presenta el boom en los estudios teóricoconceptuales sobre las ciudades y las RR.II. La mayoría de ellos, con el objetivo en común de entender la interacción entre la gobernanza global y las ciudades (globales) ${ }^{9}$, mientras que otros se han centrado en aspectos sobre la importancia de la identidad y su influencia a nivel exterior ${ }^{10}$. Así, hay quienes sostienen que las ciudades deberían ser tratadas como si fuesen auténticos agentes/actores (Acuto 2010, 2013a, 2013b, 2013c, 2013d; Lara, 2015), intentando demostrar a través del estudio de casos el potencial de "agencia" de las ciudades mundiales/globales, términos introducidos por Friedmann (1986) y Sassen

9 Amen, McCarney, Toly y Segbers (2011); Derudder, Hoyler, Taylor y Witlox (2012); Acuto (2013); Bouteligier (2013); Barber (2013); Curtis (2011).

Parker (2011); Bell y De-Shalit (2011). 
(1991) para referirse a aquellas ciudades que el capital global utiliza como puntos de base en la organización espacial y la articulación de la producción y los mercados.

Para Vilma Petrásh (1998), la gran aportación del enfoque de la ciudad global a las RR.II. es la difuminación de las escalas de estudio, lo que implica que estos territorios pueden desarrollar estrategias dentro de la gobernanza global multinivel, a saber con la intención de apelar y estimular también a comunidades académicas ubicadas en el campo más amplio de las ciencias sociales para que coadyuven de manera consciente y comprometida a producir conocimiento pertinente al eje teórico interpretativo "local(inter/trans)nacional-global" y a la construcción de las RR.II. como disciplina de cruce o transdisciplina sociocéntrica. Conocimiento, por ejemplo, que permita emprender análisis e investigaciones en el ámbito de las RR.II. afines a la que realizara Saskia Sassen en el ámbito de la sociología urbana respecto a la ciudad global, en la cual ella logró dilucidar mediante un denso aparataje empírico las relaciones, el cuadro de mediaciones y las contradicciones entre lo local y lo global, y su impacto en promover dispersiones, reconfiguraciones y neoconfiguraciones socioterritoriales (pp. 125-126).

Para Acuto, la importancia de incluir en el estudio de las RR.II. a las ciudades globales va más allá de una moda de insertar términos de la sociología urbana o el urbanismo en la disciplina o de una nueva conciencia de lo urbano. Ya que como lo señalan Mark Amen et al. (2011), las ciudades "son cada vez más decisivas en la gobernanza global" (p. 3).

Desde el enfoque urbanocéntrico (city-centric). Esta perspectiva considera que la producción de tecnología de punta y los altos salarios conforman un proceso económico en las ciudades que genera zonas centrales (core-making) haciendo posible la configuración de la red de ciudades (city network). El poder que ejercen determinadas prácticas de ciudad (city practices) para modelar las economías según sus propias necesidades, conforma espacios dependientes en los que la producción con tecnología obsoleta y bajos salarios va configurando una periferia. (Taylor, 2010, p. 151)

Para Simon Curtis (2011), detrás de la obra de P. Taylor

Está la convicción ontológica que las ciudades no deben ser vistos simplemente como lugares limitados - autocontenidos-, sino como procesos continuos de flujos de distinta índole. Esta posibilidad ontológica es una de las rutas de la vinculación de los estudios de las ciudades globales y la teoría de RR. II. (2010: 10). Al mismo tiempo: ya no pueden contener el proceso de acumulación de capital y la urbanización dentro 
de sus fronteras. Se convierten tanto en cómplices de este re-escalamiento y a su vez están más limitados por ello, así las ciudades globales y sus regiones surgen como el territorio fundamental de la globalización económica. (p. 18)

En otras palabras: "Hoy en día es posible que las ciudades mundiales estén empezando a alcanzar algunas formas nuevas de independencia respecto a los Estados territoriales, lo que las convierte en algo muy relevante para nuestra geografía política” (Taylor, 1995). Con ello no queremos decir que los Estados territoriales están a punto de desaparecer, sino que las ciudades mundiales se están convirtiendo en un nuevo locus de poder e interactuarán con los Estados de forma distinta (Taylor y Flint, 2002, p. 360). Desde otra perspectiva, ya no hay

Un centro de gravedad histórico que defina la suerte universal. Hoy se registran multitud de centros de gravedad, es decir, las ciudades. Pero ¿todas? No necesariamente. Ser un centro de gravedad, por simple y elemental que ello sea, requiere de un cierto grado de fungibilidad, de capacidad para transformar cualitativamente recursos y conductas políticas, financieras y culturales, de tal forma que la condición original se vea alterada sensiblemente. (Torrijos, 2009, p. 359)

Para Gerardo del Cerro (2004), esta dinámica provee una actuación en red:

Tenemos, por lo tanto, ciudades inscritas en redes que funcionan como nodos en la producción y reproducción de tales redes y de la materia, energía, capital e información que transmiten. Si la globalización es una red, las ciudades son también redes de relaciones, estructuras y territorios. $\mathrm{Y}$ en este contexto, en lugar de considerar la agencia del individuo aislado, lo que proponemos son las prácticas de los actoresen-redes. (p. 209)

Es en este punto donde la agencia de las ciudades (mundiales y/o globales) a través de sus redes comienza a ser vista como central para entender su influencia actual sobre otros actores. Considerando los siguientes puntos:

La agencia opera de forma diferente entre lugares e incluso de forma diferente dentro del mismo lugar en tiempos diferentes. [...] Agencia y estructura pueden verse así como un todo analítico, una unidad integrada de procesos reticulares espaciotemporales constituida por nodos y flujos. 
La noción de red nos lleva por encima de las distinciones tradicionales en ciencias sociales entre micro y macro procesos [...] En lugar de tener que escoger entre una perspectiva local y una global, la noción de red nos permite pensar la globalización como un proceso continuamente localizado.

En vez de oponer la agencia a la estructura, simplemente observamos cómo un elemento dado se convierte en relevante mediante el número de conexiones de que forma parte y cómo pierde tal relevancia al perder sus conexiones. (Del Cerro, 2004, p. 209)

\section{La ciudad global en la teoría de las RR.II.}

Los autores que más han escrito sobre las ciudades globales son Michele Acuto y Simon Curtis. Ellos siguen el enfoque holístico de la teoría del actor-red (Actor Network Theory, ANT - en castellano también conocida como sociología de la traducción u ontología del actante-rizoma-) desde la perspectiva de Bruno Latour, Michael Callon y John Law, que tienen como objetivo reensamblar lo social ${ }^{11}$. Por un lado, con la intención de incluir el estudio de las ciudades globales en las RR.II. y cómo estas forman colectivos sociales que impactan en la política mundial - como actores - y la gobernanza global —como actantes-; y por el otro, con relación a las transformaciones históricas, la teoría del ensamble "permite a los especialistas rastrear cómo se desmontan —o desensamblany son reconstruidas las capacidades e instituciones de una era histórica, y como éstas producen nuevas capacidades e instituciones" (Curtis, 2011, p. 16) en este caso durante el fenómeno histórico de la globalización.

La justificación más importante para entender el estudio de las ciudades (globales) desde las RR.II. es un artículo de Simon Curtis de 2011 "Global cities and the transformation of the International System". El autor sostiene que "cualquier intento de atribuir a las ciudades la misma importancia analítica que los Estados constituye un desafío para las RR. II., ya que estas se asientan sobre el presupuesto ontológico de que el Estado soberano opera en un sistema internacional anárquico" (Curtis, 2011, citado por Kissack, 2013 , p. 14). A tal fin, "es imperativo desarrollar bases ontológicas y epistemológicas para entender el papel de las ciudades globales en el sistema internacional" (Curtis, 2011, citado por Acuto, 2013a, p. 7). Su argumento central es:

Las ciudades no van a tomar el lugar de los Estados, pero los cambios en la naturaleza de las ciudades y los Estados en la coyuntura contemporánea forman parte de los

11 Sobre la teoría ANT, revisar Latour (2005). 
mismos procesos de re-escalamiento de la relación entre los espacios local, nacional y global. Así, el surgimiento de las ciudades globales es una manifestación de los procesos actuales de transformación que operan en el sistema internacional. (Curtis, 2010, p. 23)

En su libro Global Cities, Governance and Diplomacy. The Urban Link, Acuto (2013a) identifica las grandes limitaciones de la academia de RR.II. en lo relativo al estudio de las ciudades:

- No ha ideado de manera efectiva marcos teóricos para descubrir fragmentos del urbanismo y/o la geografía que puedan ser traducidos en consideraciones teóricas desde las RR.II.

- Por lo general, carece de una apreciación - productiva y progresiva - de la activa participación de las ciudades en la política mundial y su influencia directa que tienen en la gobernanza global.

- Cuando dicha participación e influencia se ha insinuado, hasta este momento no se han desarrollado análisis sobre los orígenes e impactos de dicha capacidad diplomática (p. 9).

Para resolver la primera limitación, al implementar la perspectiva sociológica del actorred que está ampliamente difundida en el urbanismo, explica la importancia de estas nuevas posturas. Respecto al segundo punto, asume la agencia de las ciudades globales, concepto acuñado en las disciplinas de la sociología urbana y la geografía política, para entender sus articuladores principales en la gobernanza global analizadas como "actantes", "actores" y "red de redes". Para probar la influencia de la ciudad global — the urban link-, según los postulados del ANT y el reensamblaje, analiza el caso del Cities Climate Leadership Group, o C40, como el articulador central de la gobernanza global en el tema del cambio climático.

Lo más importante de su postura es que discute sobre la agencia de las ciudades globales y sus redes transnacionales, donde el estudio de las ciudades y su agencia desde las RR.II. pasan por un impasse o han sido analizados a través de callejones sin salida (culs de sac): 1) las ciudades no son repúblicas miniatura; 2) las ciudades no son personas. Para resolver estos problemas de agencia de la ciudad ofrece tres retos analíticos: 
Las ciudades globales están incrustadas en la gobernanza global, por lo tanto, no pueden ser estudiadas como entidades separadas.

El surgimiento de las ciudades globales como actores diplomáticos en los asuntos internacionales no debe ocultar los procesos locales que se producen, pero tampoco mantener un significado puramente metafórico.

En sentido literal, los actores internacionales no son necesariamente "personas" (Acuto, 2013a, p. 58).

\section{Reflexión teórica de las ciudades como agentes en la gobernanza global multinivel}

El enfoque de la paradiplomacia ha permitido el estudio de la acción de los actores no estatales -incluidos los gobiernos subestatales - desde las RR.II., pero es insuficiente para el análisis propio de las ciudades; es por ello que la perspectiva teórico-metodológica de la ciudad global nos permite dilucidar la agencia de este tipo de lugares en la gobernanza global multinivel, gracias a su trabajo en red para definir nuevos regímenes internacionales, su capacidad de influencia política y financiera en el orden global, así como su capacidad de agencia en la gobernanza global multinivel, pero no abarca todo el espectro de las ciudades que no son "globales" y que están insertadas en el medio internacional. El mayor mérito de ambas perspectivas y sus investigaciones están en el intento teórico-metodológico para ayudarnos a cambiar el prisma y conseguir observar a la ciudad como actor y agente, en lugar de ver al Estado como el único que realiza estas funciones. Donde la paradiplomacia reafirma la importancia de los actores no estatales, y la ciudad global permite diluir la escala de análisis de las relaciones internacionales.

No obstante, al centrarse en las unidades subestatales/subnacionales, así como en las ciudades mundiales/globales, la pregunta que se plantea es: ¿qué pasa con las múltiples ciudades que no cumplen las características de ciudad mundial/global, pero que también están experimentando los procesos de cambio a partir de la retroalimentación entre globalización/urbanización? Esto conduce a la reflexión de que más bien habría que identificar qué nivel formal dinamiza los procesos de comunicación de la ciudad en el contexto global. Pues, de ese modo, contribuye directamente en la retroalimentación entre la globalización y la urbanización: se trata del gobierno de la ciudad, "por eso en muchos 
estudios desde la paradiplomacia se habla más del gobierno local o no central que de todo el espectro entendido como ciudad (territorio, identidad, población, stakeholders, etc." (Lara, 2015, p. 184), cuando debe de observarse como un sujeto completo de investigación e intervención en las RR.II.

A manera de consideraciones finales, se describen las características fundamentales de las nuevas perspectivas teórico-metodológicas para analizar las ciudades desde la gobernanza global multinivel sin necesidad de presentarse como mundiales/globales, lo que permite abrir el estudio a fenómenos relativamente subestimados desde las RR.II.

\subsection{Política pública sobre la internacionalización de las ciudades}

Desde la perspectiva de la gobernanza global es su relación con la gobernanza urbana. La inserción de la ciudad en el medio internacional, entendida como una política pública, es uno de los nuevos enfoques de estudio. Aquí, la política de inserción internacional es una política exterior. En este sentido, existen "dos formas de apreciar la política exterior. Por un lado está la práctica convencional: grupos de interés, multinacionales, organizaciones internacionales y gobiernos. Por otro lado está la gente, el juego de identidades, el escenario amplio e interconectado de las ciudades" (Torrijos, 2009, p. 362). Ya que

la mayoría de gobiernos locales realizan actividades en el exterior sin que éstas se enmarquen en un planteamiento estratégico, estén concebidas de manera integral, sean objeto de una planificación rigurosa de los recursos necesarios ni hagan participar a los actores locales y a la ciudadanía. (Malé, Cors y Sanz, 2014, p. 49)

Se parte de la idea de que las ciudades "se han convertido en actores importantes en la conformación de la política global, ayudando a forjar nuevos patrones de relaciones transnacionales" (Engelke, 2013, p. 1), ya que

son el sitio donde se observan las formas de gobierno, a través de los instrumentos de política pública. Pero también son los sitios donde se presenta la intersección entre la gobernanza y el gobierno del día a día de las personas (Amén et al., 2011, p. 3) 
Aquí se asume a la gobernanza como "la elaboración de políticas públicas que impliquen a diversos actores además del Estado y que puedan relacionarse constantemente con otras esferas además de lo público" (Marx, 2010, p. 32).

\subsection{Liderazgos locales como promotores de la inserción internacional}

Se ha dejado de lado por mucho tiempo el estudio de los liderazgos locales con la intención de sobresalir, a nivel exterior, su capacidad de gestión y de visibilizar su ciudad a nivel internacional.

Los líderes de la ciudad son con frecuencia un componente, pero casi nunca el objeto de investigación de una gran parte de los estudios, informes, columnas y colecciones que se centran en ilustrar la naturaleza cambiante de la gobernanza urbana y los cambios complejos que sustentan la gestión de la ciudad (Acuto, 2013c, p. 484; Acuto, 2014).

Estos liderazgos locales en la gobernanza global generan una diplomacia de los alcaldes, mediante el desarrollo de funciones centrales o

actividades que tienen una influencia catalítica en la gobernanza global [...] 1) Construcción y/o promoción de regímenes internacionales; 2) Hibridación de la gobernanza; 3) Emprendurismo diplomático; 4) Mediación y función normativa; e 5) Influencia e impacto en los asuntos internacionales del día a día. (Acuto, 2013c, pp. 481 y 487$)$

\subsection{Estatus de la ciudad en el Derecho Internacional}

Los estudios de las ciudades como posibles sujetos de Derecho Internacional y su influencia en la gobernanza global en la última década han ido en aumento. "Para los amantes acérrimos del orden jurídico internacional, de los preceptos constitucionales, siempre subsistirá la idea de negarle el estatus de actor internacional a las ciudades (categoría sociológica que, no obstante, el derecho incorpora cada vez más en su discurso)" (Torrijos, 2009, p. 368). Esto se debe a que "sus procesos legislativos están comenzando a tener un impacto significativo en los procesos de la gobernanza global" 
(Aust, 2015, p. 256). Con relación a las ciudades, Helmut P. Aust (2015) argumenta que el Derecho Internacional debe cumplir dos funciones en este sentido: reconocimiento y contestación.

A través del "incipiente Derecho Local Municipal” ha puesto en la mira los estudios realizados en las últimas dos décadas sobre la ciudad como posible sujeto de Derecho Internacional (Beltrán de Felipe, 2010). Aust señala que "si bien es sólo un experimento intelectual, potencialmente presagia la consecuencia de la aparición de las ciudades como actores internacionales [entendidas como sujetos de derecho internacional] donde se tendría que observar el creciente papel de las ciudades en los procesos normativos internacionales, ello provocaría un desafío a la estructura del Derecho Internacional basada en el 'consentimiento'. Aunque sólo sea por la multiplicación de los actores involucrados, sería difícil mantener el principio sobre el 'consentimiento"' (Aust, 2015, p. 276). Con la intención de consolidar una autonomía internacional y crear un nuevo derecho local y/o municipal internacional.

\subsection{Construcción de la identidad de la ciudad para su inserción internacional}

Al igual que el individuo, los grupos étnicos, las colectividades y el Estado-nación, las unidades subestatales, las ciudades también tienen identidad o identidades propias. Vicente Torrijos (2009) considera que es más que una identidad, "es una metaidentidad, un corpus imperfecto que colecciona en sí mismo y al mismo tiempo, lo que puede definir a un grupo étnico por separado: es decir, lenguas, confesiones, nacionalidades, razas en sentido bruto, o deformadas por las combinaciones multifactoriales entre tales variables" (pp. 366-367).

Las ciudades tienen la posibilidad de construir su propia identidad. Algunos autores la definen como localismo (López, 1993), ciudadanía (Holston y Appadurai, 1996), lugarización (González Cruz, 2001), patriotismo urbano (Vainer, 2003), patriotismo cívico (Borja y Castells, 2006) o autorrepresentación, para diferenciarse de las otras ciudades, pero también la de contrastar sus similitudes. Las identidades son, a final de cuentas, relaciones entre un actor y otro. En este caso, entre una ciudad y otra. 


\subsection{Difusión de la imagen de la ciudad para el reconocimiento global}

Es en este momento cuando la difusión de la identidad de la ciudad a nivel global es central para consolidarla al interior de la misma. La o las identidades están en constante construcción, ya que

las identidades son las bases de los intereses. Los actores no tienen una "agenda" de intereses que transportan consigo independientemente del contexto social en el que estén; lo que ocurre es que los actores definen sus intereses dentro del mismo proceso en el que se definen las situaciones. (Wendt, 2005, p. 8)

Los lugares, en este caso las ciudades, "han sentido siempre la necesidad de diferenciarse entre sí con el fin de afirmar su individualidad y sus características distintivas en la búsqueda de diversos objetivos económicas, políticas y socio-psicológicos" (Ashworth y Kavaratzis, 2010, p. 1).

En otras palabras,

cada ciudad, y sobre todo las más importantes, tienen una imagen consolidada: Ciudad del arte, ciudad bella, ciudad ordenada, ciudad eficiente, ciudad mágica, ciudad rica, ciudad vibrante son cualidades a menudo tan arraigadas en el imaginario colectivo que marcan el destino de la ciudad. (Amendola, 2000, p. 285)

Así, las ciudades del siglo XXI también tienen su "ethos competitivo" (Ward, 2005).

\section{Referencias}

Acuto, M. (2010). Global Cities: Gorillas in our Midst. Alternatives: Global, Local, Political, $4(35), 425-448$.

Acuto, M. (2013a). Global Cities, Governance and Diplomacy, The Urban Link. London-New York: Routledge. 
Acuto, M. (2013b). The geopolitical dimension of the global city. En M. Acuto y W. Steele (Eds.), Global City Challenges, Debating a Concept, Improving the Practice (pp. 170187). Basingstoke: Palgrave.

Acuto, M. (2013c). City Leadership in Global Governance. Global Governance: A Review of Multilateralism and International Organizations, 3(19), 481-498.

Acuto, M. (2013d). The New Climate Leaders? Review of International Studies, 4(39), 835-857.

Acuto, M. (2014). If mayors ruled the world: dysfunctional nations, rising cities. International Affairs, 2 (90), 461-462.

Aldecoa, F. y Keating, M. (1999). Paradiplomacy in Action: The Foreign Relations of Subnational Governments. London: Cass Publishers.

Amen, M., McCarney, P., Toly, N. y Segbers, K. (Eds.) (2011). Cities and Global Governance: New Sites for International Relations. London: Routledge.

Amendola, G. (2000). La Ciudad Postmoderna. Madrid: Celeste Ediciones.

Aranda, G., Ovando, C. y Corder, A. (2010). Experiencias paradiplomáticas en la región de Tarapacá y su proyección subregional. Estudios Internacionales, 165, 33-74.

Ashworth, G. y Kavaratzis, M. (Eds.) (2010). Towards Effective Place Brand Management Branding European Cities and Regions. Cheltenham-Northampton: Edward Elgar.

Aust, H. (2015). Shining Cities on the Hill? The Global City, Climate Change, and International Law. European Journal of International Law, 1(26), 255-278.

Bekemans, L. (2008). Multi-Level Governance and the EU in a Global Context: Some Introductory Reflections. Ateliers for the Committee of the Regions. Consultado 15 de abril 2013.

Beltrán de Felipe, M. (2010). Las ciudades y la globalización: tendencias y problemas en el Incipiente Derecho Local Internacional. En M.A. Martín y C. Oddone (Eds.), Las ciudades y los poderes locales en las relaciones internacionales contemporáneas (pp. 83108). Granada: UIM.

70 Si Somos Americanos. Revista de Estudios Transfronterizos 
Biersteker, T.J. (2009). Global Governance. En M. Dunn y V. Maurer (Eds.), The Routledge Handbook of Security Studies (pp. 439-451). New York and London: Routledge Publishers.

Borja, J. y Castells, M. (2006). Local y Global. La Gestión de las ciudades en la era de la información ( $2^{\mathrm{a}}$ ed.). México, D.F.: Taurus.

Bursens, P. y Deforche J. (2010). Going Beyond Paradiplomacy? Adding Historical Institutionalism to Account for Regional Foreign Policy Competences. The Hague Journal of Diplomacy, 1-2(5), 151-171.

Cerqueira, O. (2013). La Comunidad Andina de Naciones y la Unión Europea frente a la dinámica global. La Rioja: Siníndice Editorial.

Cornago, N. (1996). Acción exterior y paradiplomacia: la proyección internacional de los meso gobiernos (Tesis para obtener el grado de doctor en Estudios Internacionales). Universidad del País Vasco, Leioa.

Cornago, N. (2010). On the Normalization of Sub-State Diplomacy. The Hague Journal of Diplomacy, 1-2(5), 11-36.

Cornago, N. (2016). Diplomacia como heterología: pluralismo social y múltiples mediaciones institucionales en la frontera. En S. González, N. Cornago y C. Ovando (Eds.), Relaciones Transfronterizas y Paradiplomacia en América Latina. Aspectos teóricos y estudio de caso (pp. 17-45). Santiago: RIL editores.

Criekemans, D. (2010). Regional Sub-state Diplomacy from a Comparative Perspective: Quebec, Scotland, Bavaria, Catalonia, Wallonia and Flanders. The Hague Journal of Diplomacy, 1-2(5), 37-64.

Curtis, S. (2011). Global Cities and the Transformation of the International System. Review of International Studies, 4(37), 1923-1947.

Del Arenal, C. (2009). Mundialización, creciente interdependencia y globalización en las relaciones internacionales. En AA.VV, Cursos de Derecho Internacional y Relaciones Internacionales de Vitoria-Gasteiz, 2008 Vitoria-Gasteizko Nazioarteko Zuzenbide eta Nazioarteko Harremanen Ikastaroak. Recuperado de http://www.ehu.eus/ cursosderechointernacionalvitoria/ponencias/pdf/2008/2008_4.pdf 
Del Cerro, G. (2004). Ciudades y globalización: un enfoque teórico. Revista Española de Sociología, 4, 199-218.

Diez de Velasco, M. (2013). Instituciones de Derecho Internacional Público (18 a ed.). Madrid: Tecnos.

Duchacek, I. (1986). The Territorial Dimension of Politics: Within, Among and Across Nations. Boulder: Westview.

Duchacek, I. (1990). Perforated sovereignties: towards a typology of new actors in international relations. En H.J. Michelmann y P. Soldatos (Eds.), Federalism and international relations: the role of subnational units (pp. 1-32). New York: Oxford University Press.

Duran, M. (2012). Bridging the gap between self and other? Mediterranean Paradiplomacy as Homo-diplomacy. ECPR Antwerp Joint Research Sessions. Workshop The Transformation of Foreign Policy and Diplomacy

Duran, M. (2013). Sub-State Diplomacy as a Motor of Reterritorialization? The Case of the Mediterranean Region (Tesis para obtener el grado de doctor). Universidad de Amberes-Politieke Wetenschappen aan de Universiteit Antwerpen, Bélgica.

Engelke, P. (2013). Foreign Policy for an Urban World: Global Governance and the Rise of Cities. Atlantic Council. Recuperado de http://www.atlanticcouncil.org/publications/ issue-briefs/foreign-policy-for-an-urban-world-global-governance-and-the-rise-ofcities

García i Segura, C. (1996). La actividad exterior de las entidades políticas subestatales. Revista de Estudios Políticos, 91, 234-264.

González Cruz, F. (2001). Localization. Mérida: Fondo Editorial Universidad Valle del Momboy.

Grasa, R. (2000). Globalització, sobirania i interdependencia. En E. Fossas (Ed.), Les transformacions de la sobirania i el futur polític de Catalunya (pp. 215-245). Barcelona: Proa.

Hocking, B. (1993). Localizing Foreign Policy, Non-Central Governments and Multilayered Diplomacy. London: St. Martin's Press.

72 Si Somos Americanos. Revista de Estudios Transfronterizos 
Holston, J. y Appadurai, A. (1996). Cities and Citizenship. Public Culture, 8, 187-204.

Kooiman, J. (2004). Gobernar en gobernanza. Revista Instituciones y Desarrollo, 16, 171-194.

Lara, R. (2015). La construcción de ciudades modelo y su inserción internacional los casos de ciudad de México, Singapur y Bilbao (Tesis para obtener el grado de doctor en Estudios Internacionales). Universidad del País Vasco, Leioa.

Lecours, A. (2008). Political Issues of Paradiplomacy: Lessons from the Developed World. Discussion papers in diplomacy. Den Haag: Institute Clingendael.

López, Á. (1993). Memoria y nacionalismo. Trabajo presentado en el congreso Los nacionalismos. Seminario de Investigación para la Paz, Departamento de Educación y Cultura del Gobierno de Aragón, Zaragoza.

Luna Pont, M. (2010). Perspectivas teórico-conceptuales de las ciudades y los poderes locales dentro de la dimensión subnacional en las Relaciones Internacionales. En M.A. Martín y C. Oddone (Eds.), Las ciudades y los poderes locales en las relaciones internacionales contemporáneas (pp. 71-78). Granada: UIM.

Luna Pont, M. (2011). Las Relaciones Internacionales subnacionales desde la Teoría de las Relaciones Internacionales. Revista TIP (Trabajos de Investigación en Paradiplomacia), 1(1), pp. 64-83. Recuperado de http://paradiplomacia.org/upload/imagenes/9ee0b5c5 4b06c7fb6d8fed8b79ef6dd3tip_a\%C3\%B1o1no1.pdf

Malé, J.P, Cors, C. y Sanz, V. (2014). Internacionalización del gobierno local. Una política pública en construcción [versión eBook]. Recuperado de https://www.proyectoallas. net/documents/161716/190300/CUADERNO+AL-LAs+02.pdf/cdfb9c7a-7ebe-4ea3$9 \mathrm{c} 67-2 \mathrm{c} 84383 \mathrm{c} 74 \mathrm{ab}$

Marx, V. (2010). Las ciudades y su inserción política en las relaciones internacionales. En M.A. Martín y C. Oddone (Eds.), Las ciudades y los poderes locales en las relaciones internacionales contemporáneas (pp. 25-47). Granada: UIM.

Mayntz, R. (2001). El Estado y la sociedad civil en la gobernanza moderna. Revista del CLAD Reforma y Democracia, 21. Recuperado de http://old.clad.org/portal/publicacionesdel-clad/revista-clad-reforma-democracia/articulos/021-octubre-2001/0041004 
Oddone, N. (2016). La paradiplomacia desde cinco perspectivas: reflexiones teóricas para la construcción de una comunidad epistémica. RI Revista, 2(89). Recuperado de http:// revistas.una.ac.cr/index.php/ri/article/view/9064

Paquin, S. (2010). Federalism and Multi-Level Governance in Foreign Affairs: a Comparison of Canada and Belgium. En F. Requejo (Ed.), Foreign policy of constituents units at the beginning of 21st century (pp. 161-186). Barcelona: Generalitat de Catalunya. Institut d'Estudis Autonòmics.

Paquin, S. (2005). Les actions extérieures des entités subétatiques : quelle signification pour la politique comparée et les relations internationales? Revue internationale de politique compare, 12, 129-142. Recuperado de https://www.cairn.info/revue-internationalede-politique-comparee-2005-2-page-129.htm

Petrásh, V. (1998). De lo internacional a lo global. Reconstruyendo los estudios internacionales en los tiempos de post-internacionalización: aportes para el tercer debate [en línea]. Recuperado de https://es.scribd.com/document/70946057/Vilma-E-Petrash-De-loInternacional-a-lo-Global

Philippart, É. (1997). Le comité de regions confronté à la paradiplomatie des règions de l'Union Européenne. En J. Bourrinet J. (Ed.), Le Comité des Regions de l'Union Européenne (pp. 6-13). Paris: Editions économica.

Salomón, M. (2007). La Acción Exterior de los Gobiernos Subnacionales y el Análisis de Políticas Exteriores. Trabajo presentado en el congreso Primer Encontro Nacional da ABRI Política Externa, Belo Horizonte.

Simmerl, G. (2011). A Critical Constructivist Perspective on Global Multi-Level Governance. Discursive Struggles among Multiple Actors in a Globalized Political Space. Manuscrito inédito, Freie Universität Berlin, Berlín.

Soldatos, P. (1990). An Explanatory Framework for the Study of Federated States as ForeignPolicy Actors. En H.J. Michelmann y P. Soldatos, Federalism and international relations: the role of subnational units (pp. 33-53). New York: Oxford University Press.

Taylor, P.J. (2010). La red de ciudades mundiales y el planeta de los barrios pobres: acceso y exclusión en la globalización neoliberal. En M. Alfie, I. Bueno, C. Pérez y M. Tamayo (Eds.), Sistema Mundial y nuevas geografías (pp. 147-174). México, D.F.: UIA-UAM.

74 Si Somos Americanos. Revista de Estudios Transfronterizos 
Taylor, P. y Flint, C. (2002). Geografía Política: economía-mundo, Estado-nación y Localidad. Madrid: Trama editorial.

Torrijos, V. (2009). Política exterior y relaciones internacionales. Bogotá: Universidad del Rosario.

Vainer, C. (2003). Pátria, empresa e mercadoria. Notas sobre a estrategia discursiva do planeamiento estratégico urbano. En O. Arantes, C. Vainer y E. Maricato (Eds.), A ciudade do pensamento único: desmanchando consensos ( $3^{\mathrm{a}}$ ed., pp. 75-103). Petrópolis: Vozes.

Van der Pluijm, R. y Melissen J. (2007). City Diplomacy: The Expanding Role of Cities in International Politics [version electrónica]. Recuperado de http://www.clingendael.nl/ publication/city-diplomacy-expanding-role-cities-international-politics

Ward, S.V. (2005). Selling Places, The Marketing and Promotion of Towns and Cities, 1850-2000 [versión e-book]. Recuperado de http://www.tandfebooks.com/doi/ view/10.4324/9780203976746

Wendt, A. (2005). La anarquía es lo que los Estados hacen de ella. La construcción social de la política de poder. Revista Académica de Relaciones Internacionales, 1. Recuperado de http://www.relacionesinternacionales.info/ojs/article/view/6.html

Zapata, E. (2007). Manual práctico para internacionalizar la ciudad. Guía para la acción exterior de los gobiernos locales y la cooperación descentralizada Unión EuropeaAmérica Latina. Volumen 1 [versión electrónica]. Recuperado de http://observ-ocd. org/es/manual-practico-para-internacionalizar-la-ciudad 